VOL. $62(2000)$ [389-401]

\title{
MEASURE OF WEAK NONCOMPACTNESS AND REAL INTERPOLATION OF OPERATORS
}

\author{
Andrzej Kryczka, Stanislaw Prus and Mariusz Szczepanik
}

\begin{abstract}
A new measure of weak noncompactness is introduced. A logarithmic convexity-type result on the behaviour of this measure applied to bounded linear operators under real interpolation is proved. In particular, it gives a new proof of the theorem showing that if at least one of the operators $T: A_{i} \rightarrow B_{i}, i=0,1$ is weakly compact, then so is $T: A_{\theta, p} \rightarrow B_{\theta, p}$ for all $0<\theta<1$ and $1<p<\infty$.
\end{abstract}

\section{INTRODUCTION}

In 1960, Krasnoselskii [20] proved that under the hypotheses of the Riesz-Thorin interpolation theorem (that is, if $T$ is a linear operator such that $T: L_{p_{i}} \rightarrow L_{q_{i}}$ is bounded for $i=0,1$ where $\left.p_{i}, q_{i} \in[1, \infty]\right)$ and the additional assumption that $T$ : $L_{p_{0}} \rightarrow L_{q_{0}}$ is compact and $q_{0}<\infty$, it follows that $T: L_{p} \rightarrow L_{q}$ is also compact. Here $1 / p=(1-\theta) / p_{0}+\theta / p_{1}, 1 / q=(1-\theta) / q_{0}+\theta / q_{1}$ and $0<\theta<1$.

This has initiated a series of theorems which refer to the question whether in the above theorem the pairs $\left(L_{p_{0}}, L_{p_{1}}\right),\left(L_{q_{0}}, L_{q_{1}}\right)$ and the spaces $L_{p}, L_{q}$ can be replaced by pairs of Banach spaces $\left(A_{0}, A_{1}\right),\left(B_{0}, B_{1}\right)$ and real interpolation spaces $A_{\theta, p}, B_{\theta, p}$. More generally, we can ask if $T$, viewed as a map from $A_{\theta, p}$ to $B_{\theta, p}$, inherits any compactness properties which it may possess as an element of $L\left(A_{i}, B_{i}\right)$.

Since the works of Lions and Peetre [21], several authors have obtained results of different degrees of generality. Finally, Cwikel [15] showed that if $T: A_{0} \rightarrow B_{0}$ is compact and $T: A_{1} \rightarrow B_{1}$ is bounded, then $T: A_{\theta, p} \rightarrow B_{\theta, p}$ is also compact for all $0<\theta<1$ and $1<p<\infty$. The similar problem for weak compactness properties of $T$ was studied as well. Here a key result is due to Beauzamy [8], who proved that if the embedding $I: A_{0} \cap A_{1} \rightarrow A_{0}+A_{1}$ is weakly compact, then so is $I: A_{\theta, p} \rightarrow A_{\theta, p}$. Next, Heinrich [18] extended this result to closed operator ideals. Other generalisations of Beauzamy's result are due to Aizenstein and Brudnyi (see the book [9]), and to Maligranda and Quevedo [22] (see also Mastylo [23]) who established the following: if $0<\theta<1,1<p<\infty$ and $T: A_{0} \cap A_{1} \rightarrow B_{0}+B_{1}$ is weakly compact, then so is $T: A_{\theta, p} \rightarrow B_{\theta, p}$. In particular, this is the case if $T: A_{0} \rightarrow B_{0}$ or $T: A_{1} \rightarrow B_{1}$ is weakly compact.

Received 31st January, 2000

Copyright Clearance Centre, Inc. Serial-fee code: 0004-9727/00 \$A2.00+0.00. 
The Riesz-Thorin theorem gives also a logarithmically convex estimate for the norms of an interpolated operator. This motivated some authors to find quantitative versions of the above-mentioned results on compact and weakly compact operators. The main tools in such investigations are measures of noncompactness and weak noncompactness. Measures of noncompactness appear in various contexts, so it is convenient to define many different measures (see [1]). Here let us mention only the Hausdorff and separation measures. In contrast, very few measures of weak noncompactness can be found in the literature. Most authors use the measure of weak noncompactness $\omega$ introduced by De Blasi [16], which can be seen as a counterpart of the Hausdorff measure of noncompactness. De Blasi's measure was successfully applied to operator theory and to the theory of differential and integral equations (see [5] and the references given there). The measure $\omega$ was also used by Aksoy and Maligranda [3] in order to obtain a quantitative version of the theorem on real interpolation of weakly compact operators. (Similar results for the Hausdorff measure of noncompactness were earlier proved in [27].) Recently the thorough study of this kind of estimates for operators under real interpolation was undertaken by Cobos, Fernández-Martínez, Manzano and Martínez in the series of papers $[10,11,12,13,14]$. In particular, some essential restrictions on interpolation of De Blasi's measure are indicated (see $[12,14])$.

In this paper we introduce a new measure of weak noncompactness $\gamma$, which can be treated as a counterpart of the separation measure of noncompactness. Its definition bases on an idea due to James [19]. In the general case, the measures $\omega$ and $\gamma$ are not equivalent. The measure $\gamma$ appeals directly to the norm topology, while in the definition of $\omega$ the weak topology is involved. This seems to determine a more geometric character of $\gamma$. However, $\gamma$ can be expressed by various formulae. In particular, we show that $\gamma$ coincides with the function based on the double-limit criterion of weak compactness, which was considered by Astala and Tylli in [4]. Our main result shows that Riesz-Thorin type estimates hold for the measure $\gamma$ without any additional restrictions.

Throughout this paper, by a Banach space we mean a real one. However, the results presented here can be easily restated and proved for complex Banach spaces. We denote the open unit ball of a Banach space $X$ by $\mathrm{B}_{X}$ and its closure by $\overline{\mathrm{B}}_{X}$. Furthermore, conv $A$ stands for the convex hull of a set $A \subset X$ and the closure of conv $A$ will be denoted by Conv $A$. For simplicity of notation we use the same letter to designate an element of a Banach space $X$ and its canonical image in the second dual $X^{* *}$. The abbreviations with the beginning $w^{*}$ refer to the weak-star topology.

\section{MEASURES OF WEAK NONCOMPACTNESS}

2.1. Measure $\gamma$ Let $\left(x_{n}\right)$ be a sequence in a Banach space $X$. We say that $\left(y_{n}\right)$ is a sequence of successive convex combinations, or scc, for $\left(x_{n}\right)$ if there exists a sequence of integers $0=p_{1}<p_{2}<p_{3}<\ldots$ such that $y_{n} \in \operatorname{conv}\left(x_{i}\right)_{i=p_{n+1}}^{p_{n+1}}$ for every $n$. Similarly, 
vectors $u_{1}, u_{2}$ are said to be a pair of $s c c$ for $\left(x_{n}\right)$ if $u_{1} \in \operatorname{conv}\left(x_{i}\right)_{i=1}^{p}, u_{2} \in \operatorname{conv}\left(x_{i}\right)_{i=p+1}^{\infty}$ for some $p \in \mathbb{N}$.

The following theorem, motivated by [25], will play a significant role in the sequel.

THEOREM 2.1. Let $\left(x_{n}\right)$ be a bounded sequence in a Banach space $X$. For every $\varepsilon>0$ there exists a sequence $\left(y_{n}\right)$ of scc for $\left(x_{n}\right)$ such that if $u_{1}, u_{2}$ and $v_{1}, v_{2}$ are any pairs of scc for $\left(y_{n}\right)$, then $\left|\left\|u_{1}-u_{2}\right\|-\left\|v_{1}-v_{2}\right\|\right| \leqslant \varepsilon$.

Proof: Let $A_{n}=\operatorname{Conv}\left(x_{i}\right)_{i=n}^{\infty}$ for $n \in \mathbb{N}$. Assume first that $\bigcap_{n=1}^{\infty} A_{n} \neq \emptyset$. Then we can find a convergent sequence of $s c c$ for $\left(x_{n}\right)$ and, by ignoring a finite number of terms if necessary, we get a sequence as required. Suppose now that $\bigcap_{n=1}^{\infty} A_{n}=\emptyset$. Reasoning similar to that in the proof of the corollary of Theorem 2 [25] (see also [26]) gives a constant $d>0$ such that for any $\varepsilon>0$ there exists $\left(y_{n}\right)$ of $\operatorname{scc}$ for $\left(x_{n}\right)$ such that $d-\varepsilon \leqslant\left\|u_{1}-u_{2}\right\| \leqslant d$ for any pair $u_{1}, u_{2}$ of $s c c$ for $\left(y_{n}\right)$. Clearly, $\left(y_{n}\right)$ fulfills the assertion of the theorem.

Now, following [6], we introduce an axiomatic approach to the notion of a measure of weak noncompactness. Let $\mu$ be a real-valued function defined on the family of all bounded and nonempty subsets of a Banach space $X$. We call $\mu$ a measure of weak noncompactness on $X$, if the following conditions are satisfied for any subsets $A, B$ and $c \in \mathbb{R}:$

(1) $\mu(A)=0$ if and only if $A$ is a relatively weakly compact set;

(2) if $A \subset B$, then $\mu(A) \leqslant \mu(B)$;

(3) $\mu(\operatorname{Conv} A)=\mu(A)$;

(4) $\mu(A \cup B)=\max \{\mu(A), \mu(B)\}$;

(5) $\mu(A+B) \leqslant \mu(A)+\mu(B)$;

(6) $\mu(c A)=|c| \mu(A)$.

Definition 2.2: We introduce the function $\gamma$ defined for every nonempty and bounded set $A \subset X$ by the formula

$$
\gamma(A)=\sup \left\{\operatorname{csep}\left(x_{n}\right):\left(x_{n}\right) \subset \operatorname{conv} A\right\},
$$

where

$$
\operatorname{csep}\left(x_{n}\right)=\inf \left\{\left\|y_{1}-y_{2}\right\|: y_{1}, y_{2} \text { is a pair of } s c c \text { for }\left(x_{n}\right)\right\} .
$$

THEOREM 2.3. $\gamma$ is a measure of weak noncompactness on $X$.

Proof: Conditions (2), (3) and (6) are easy to check. Implications in (1) for $\gamma$ are consequences of the James theorem [19] and Mazur theorem [24] respectively.

To prove (5), let us take bounded $A, B \subset X$ and a sequence $\left(x_{n}\right) \subset \operatorname{conv}(A+B)$. For every $n \in \mathbb{N}$ there exist nonnegative $\lambda_{1}^{n}, \ldots, \lambda_{m_{n}}^{n}$, where $\sum_{i=1}^{m_{n}} \lambda_{i}^{n}=1$, and elements $u_{i}^{n} \in A$, 
$v_{i}^{n} \in B, 1 \leqslant i \leqslant m_{n}$ such that $x_{n}=\sum_{i=1}^{m_{n}} \lambda_{i}^{n}\left(u_{i}^{n}+v_{i}^{n}\right)$. Let $y_{n}=\sum_{i=1}^{m_{n}} \lambda_{i}^{n} u_{i}^{n}, z_{n}=\sum_{i=1}^{m_{n}} \lambda_{i}^{n} v_{i}^{n}$ and fix $\varepsilon>0$. By Theorem 2.1 there exists a sequence $\left(y_{n}^{\prime}\right)$ of $s c c$ for $\left(y_{n}\right)$ such that $\left|\left\|w_{1}-w_{2}\right\|-\left\|w_{1}^{\prime}-w_{2}^{\prime}\right\|\right| \leqslant \varepsilon$ for any pairs $w_{1}, w_{2}$ and $w_{1}^{\prime}, w_{2}^{\prime}$ of $s c c$ for $\left(y_{n}^{\prime}\right)$. Each term in $\left(y_{n}^{\prime}\right)$ takes the form $y_{k}^{\prime}=\sum_{i=n_{k}+1}^{n_{k+1}} \mu_{i}^{k} y_{i}$, where $\mu_{i}^{k}$ are nonnegative and $\sum_{i=n_{k}+1}^{n_{k+1}} \mu_{i}^{k}=1$. Set $z_{k}^{\prime}=\sum_{i=n_{k}+1}^{n_{k+1}} \mu_{i}^{k} z_{i}$. By applying again Theorem 2.1 we get a sequence $\left(z_{n}^{\prime \prime}\right)$ of $\operatorname{scc}$ for $\left(z_{n}^{\prime}\right)$ such that $\left|\left\|v_{1}-v_{2}\right\|-\left\|z_{1}^{\prime \prime}-z_{2}^{\prime \prime}\right\|\right| \leqslant \varepsilon$ for any pair $v_{1}, v_{2}$ of $s c c$ for $\left(z_{n}^{\prime \prime}\right)$. For such obtained $\left(z_{n}^{\prime \prime}\right)$ we can construct a sequence $\left(y_{n}^{\prime \prime}\right)$ of $s c c$ for $\left(y_{n}^{\prime}\right)$ in the similar way as we constructed $\left(z_{n}^{\prime}\right)$ for a given $\left(y_{n}^{\prime}\right)$. Then

$$
\left\|y_{1}^{\prime \prime}-y_{2}^{\prime \prime}\right\| \leqslant \operatorname{csep}\left(y_{n}^{\prime \prime}\right)+\varepsilon \quad \text { and } \quad\left\|z_{1}^{\prime \prime}-z_{2}^{\prime \prime}\right\| \leqslant \operatorname{csep}\left(z_{n}^{\prime \prime}\right)+\varepsilon .
$$

Note that $x_{1}^{\prime \prime}=y_{1}^{\prime \prime}+z_{1}^{\prime \prime}, x_{2}^{\prime \prime}=y_{2}^{\prime \prime}+z_{2}^{\prime \prime}$ form a pair of $s c c$ for $\left(x_{n}\right)$ and therefore

$$
\begin{aligned}
\operatorname{csep}\left(x_{n}\right) & \leqslant\left\|x_{1}^{\prime \prime}-x_{2}^{\prime \prime}\right\| \leqslant\left\|y_{1}^{\prime \prime}-y_{2}^{\prime \prime}\right\|+\left\|z_{1}^{\prime \prime}-z_{2}^{\prime \prime}\right\| \leqslant \operatorname{csep}\left(y_{n}^{\prime \prime}\right)+\operatorname{csep}\left(z_{n}^{\prime \prime}\right)+2 \varepsilon \\
& \leqslant \gamma(A)+\gamma(B)+2 \varepsilon .
\end{aligned}
$$

An arbitrary choice of $\varepsilon$ yields $\operatorname{csep}\left(x_{n}\right) \leqslant \gamma(A)+\gamma(B)$. Thus $\gamma(A+B) \leqslant \gamma(A)+\gamma(B)$.

To obtain (4), first we see from (2) that $\max \{\gamma(A), \gamma(B)\} \leqslant \gamma(A \cup B)$. For the opposite inequality let us choose a sequence $\left(x_{n}\right)$ in $\operatorname{conv}(A \cup B)$. Each $x_{n}$ takes the form $x_{n}=t_{n} y_{n}+\left(1-t_{n}\right) z_{n}$ for some $t_{n} \in[0,1], y_{n} \in \operatorname{conv} A$ and $z_{n} \in$ conv $B$. There exists a subsequence $\left(t_{n_{k}}\right)$ convergent to some $t \in[0,1]$. The sets $A, B$ are bounded, so $M=\sup \{\|x\|: x \in A \cup B\}<\infty$ and for arbitrarily fixed $\varepsilon>0$ we can assume that $\left|t_{n_{k}}-t\right| \leqslant \varepsilon /(M+1)$ for all $k$. Then $\left\|\left(t_{n_{k}}-t\right) y_{n_{k}}\right\| \leqslant(\varepsilon /(M+1))\left\|y_{n_{k}}\right\| \leqslant \varepsilon$ and similarly $\left\|\left(1-t_{n_{k}}\right) z_{n_{k}}-(1-t) z_{n_{k}}\right\| \leqslant \varepsilon$. It follows that $\left\|x_{n_{k}}-u_{k}\right\| \leqslant 2 \varepsilon$, where $u_{k}=t y_{n_{k}}+(1-t) z_{n_{k}}$. Since $\left(u_{k}\right)$ is a sequence in $t$ conv $A+(1-t)$ conv $B$, we have

$$
\begin{aligned}
\operatorname{csep}\left(u_{k}\right) & \leqslant \gamma(t \operatorname{conv} A+(1-t) \operatorname{conv} B) \leqslant t \gamma(A)+(1-t) \gamma(B) \\
& \leqslant \max \{\gamma(A), \gamma(B)\} .
\end{aligned}
$$

But $\operatorname{csep}\left(x_{n_{k}}\right) \leqslant \operatorname{csep}\left(u_{k}\right)+4 \varepsilon$, which shows that

$$
\operatorname{csep}\left(x_{n}\right) \leqslant \operatorname{csep}\left(x_{n_{k}}\right) \leqslant \max \{\gamma(A), \gamma(B)\}+4 \varepsilon .
$$

Finally, $\gamma(A \cup B) \leqslant \max \{\gamma(A), \gamma(B)\}$.

In the next two results we establish alternative formulae for the measure $\gamma$.

THEOREM 2.4. Let $A$ be a nonempty and bounded subset of a Banach space $X$. Then

$$
\gamma(A)=\sup \operatorname{dist}\left(x^{* *}, \operatorname{conv}\left(x_{n}\right)\right),
$$

where the supremum is taken over all sequences $\left(x_{n}\right)$ in conv $A$ and all $w^{*}$-cluster points $x^{* *} \in X^{* *}$ of a sequence $\left(x_{n}\right)$. 
Proof: Let us denote by $\gamma^{\prime}(A)$ the right-hand side of formula (2.1). In order to show that $\gamma^{\prime}(A) \geqslant \gamma(A)$ we argue as in the proof of [26, Lemma 2.1]. Indeed, for $\varepsilon>0$ let us find a sequence $\left(x_{n}\right)$ in conv $A$ such that $\gamma(A)-\varepsilon \leqslant \operatorname{csep}\left(x_{n}\right)$ and fix $x \in \operatorname{conv}\left(x_{n}\right)$. There exists $m \in \mathbb{N}$ such that if $y \in \operatorname{conv}\left(x_{i}\right)_{i=m}^{\infty}$, then $x, y$ is a pair of $s c c$ for $\left(x_{n}\right)$ and therefore $\|x-y\| \geqslant \operatorname{csep}\left(x_{n}\right) \geqslant \gamma(A)-\varepsilon$. By applying a separation theorem, we can find a functional $x^{*} \in X^{*}$ such that $\left\|x^{*}\right\| \leqslant 1$ and $x^{*}(y-x) \geqslant \gamma(A)-\varepsilon$ for all $y \in \operatorname{conv}\left(x_{i}\right)_{i=m}^{\infty}$. Let $x^{* *} \in X^{* *}$ be a $w^{*}$-cluster point of $\left(x_{n}\right)$. Then $x^{* *}\left(x^{*}\right)=\lim _{k \rightarrow \infty} x^{*}\left(x_{n_{k}}\right)$ for some subsequence $\left(x_{n_{k}}\right)$ and consequently $\left\|x^{* *}-x\right\| \geqslant\left(x^{* *}-x\right)\left(x^{*}\right)=\lim _{k \rightarrow \infty} x^{*}\left(x_{n_{k}}-x\right) \geqslant$ $\gamma(A)-\varepsilon$. This gives $\operatorname{dist}\left(x^{* *}, \operatorname{conv}\left(x_{n}\right)\right) \geqslant \gamma(A)-\varepsilon$ and finally, $\gamma^{\prime}(A) \geqslant \gamma(A)$.

The proof of the opposite inequality is a modification of reasoning in [19] (see also [17]). For each $\varepsilon>0$ there exists a sequence $\left(x_{n}\right)$ in conv $A$ and its $w^{*}$-cluster point $x^{* *} \in X^{* *}$ such that $\operatorname{dist}\left(x^{* *}, \operatorname{conv}\left(x_{n}\right)\right) \geqslant \gamma^{\prime}(A)-\varepsilon$. By a separation theorem we obtain a functional $x^{* * *} \in X^{* * *}$ such that $\left\|x^{* * *}\right\| \leqslant 1$ and $x^{* * *}\left(x^{* *}-x\right) \geqslant \gamma^{\prime}(A)-\varepsilon$ for all $x \in \operatorname{conv}\left(x_{n}\right)$. We now choose by induction a sequence of functionals $\left(x_{k}^{*}\right) \subset X^{*}$ and a subsequence $\left(x_{n_{k}}\right)$ with the following properties: $\left\|x_{k}^{*}\right\| \leqslant 1+\varepsilon$ for all $k \in \mathbb{N}$, $x_{k}^{*}\left(x_{n_{i}}\right) \geqslant x^{* * *}\left(x^{* *}\right)-\varepsilon$ if $k \leqslant i$ and $x_{k}^{*}\left(x_{n_{i}}\right)=x^{* * *}\left(x_{n_{i}}\right)$ if $i<k$. To construct these sequences, we apply the principle of local reflexivity $[17, p .33]$. By this principle, there exists $x_{1}^{*} \in X^{*}$ such that $\left\|x_{1}^{*}\right\| \leqslant 1+\varepsilon$ and $x^{* *}\left(x_{1}^{*}\right)=x^{* * *}\left(x^{* *}\right)$. Since $x^{* *}$ is the $w^{*}$-cluster point of $\left(x_{n}\right)$ we can find $n_{1} \in \mathbb{N}$ satisfying $\left|x_{1}^{*}\left(x_{n_{1}}\right)-x^{* *}\left(x_{1}^{*}\right)\right| \leqslant \varepsilon$. Let us assume now, that we have obtained the required $x_{1}^{*}, \ldots, x_{k-1}^{*}$ and $x_{n_{1}}, \ldots, x_{n_{k-1}}$. There exists $x_{k}^{*} \in X^{*}$ such that $\left\|x_{k}^{*}\right\| \leqslant 1+\varepsilon, x_{k}^{*}\left(x_{n_{i}}\right)=x^{* * *}\left(x_{n_{i}}\right)$ for $i=1, \ldots, k-1$ and $x^{* *}\left(x_{k}^{*}\right)=x^{* * *}\left(x^{* *}\right)$. We choose a number $n_{k}>n_{k-1}$ to satisfy $\left|x_{i}^{*}\left(x_{n_{k}}\right)-x^{* *}\left(x_{i}^{*}\right)\right| \leqslant \varepsilon$ for $i=1, \ldots, k$. Let us consider now a pair $u, v$ of $s c c$ for $\left(x_{n_{i}}\right)$. There exists $k>1$ such that $u \in \operatorname{conv}\left(x_{n_{i}}\right)_{i=1}^{k-1}$, $v \in \operatorname{conv}\left(x_{n_{i}}\right)_{i=k}^{\infty}$. Of course $x_{k}^{*}(u)=x^{* * *}(u)$ and $x_{k}^{*}(v) \geqslant x^{* * *}\left(x^{* *}\right)-\varepsilon$, and hence $(1+\varepsilon)\|u-v\| \geqslant x_{k}^{*}(v-u) \geqslant x^{* * *}\left(x^{* *}-u\right)-\varepsilon \geqslant \gamma^{\prime}(A)-2 \varepsilon$. From this it follows that $\operatorname{csep}\left(x_{n_{i}}\right) \geqslant\left(\gamma^{\prime}(A)-2 \varepsilon\right) /(1+\varepsilon)$, which gives $\gamma(A) \geqslant\left(\gamma^{\prime}(A)-2 \varepsilon\right) /(1+\varepsilon)$. Letting $\varepsilon \rightarrow 0$ we obtain $\gamma(A) \geqslant \gamma^{\prime}(A)$, and the proof is complete.

The measure $\gamma$ is equal to the function measuring the deviation from relative weak compactness based on the classical double-limit criterion. A function of this type was considered also in [4].

THEOREM 2.5. Let $A$ be a nonempty and bounded subset of a Banach space $X$. Then

$$
\begin{aligned}
\gamma(A)=\sup \left\{\lim _{n} \lim _{k} F_{n}\left(x_{k}\right)-\lim _{k} \lim _{n} F_{n}\left(x_{k}\right):\right. \\
\left.\left(x_{k}\right) \subset \operatorname{conv} A,\left(F_{n}\right) \subset \overline{\mathrm{B}}_{X} . \text { and the limits exist }\right\} .
\end{aligned}
$$

Proof: Let us denote by $e(A)$ the right-hand side of formula (2.2). From the proof of Theorem 2.4 it follows that there exists $a$ such that for every $\varepsilon>0$ one can find sequences $\left(x_{k}\right) \subset$ conv $A$ and $\left(F_{n}\right) \subset \overline{\mathrm{B}}_{X}$. satisfying the following: $F_{n}\left(x_{k}\right) \geqslant a-\varepsilon$ for 
$n \leqslant k$ and $F_{n}\left(x_{k}\right) \leqslant a-\gamma(A)+\varepsilon$ for $n>k$. By passing to a subsequence, if necessary, we can assume that all the limits $\alpha_{1}=\lim _{n} \lim _{k} F_{n}\left(x_{k}\right)$ and $\alpha_{2}=\lim _{k} \lim _{n} F_{n}\left(x_{k}\right)$ exist. Of course, $\gamma(A)-2 \varepsilon \leqslant \alpha_{1}-\alpha_{2}$. Hence $\gamma(A)^{n}-2 \varepsilon \leqslant e(A)$ and $\gamma(A) \leqslant e(A)$.

Let $\left(x_{k}\right) \subset \operatorname{conv} A$ and $\left(F_{n}\right) \subset \bar{B}_{X}$. be any sequences for which the limits $\beta_{1}=$ $\lim _{n} \lim _{k} F_{n}\left(x_{k}\right)$ and $\beta_{2}=\lim _{k} \lim _{n} F_{n}\left(x_{k}\right)$ exist. By Theorem 2.1 for arbitrary $\varepsilon>0$ we can find a sequence $\left(z_{k}\right)$ of $s c c$ for $\left(x_{k}\right)$ such that $\left\|z_{i}-z_{j}\right\|-\operatorname{csep}\left(z_{k}\right) \leqslant \varepsilon$ for every $i \neq j$. Then $\beta_{1}=\lim _{n} \lim _{k} F_{n}\left(z_{k}\right)$ and $\beta_{2}=\lim _{k} \lim _{n} F_{n}\left(z_{k}\right)$. Therefore $\beta_{1}-\beta_{2} \leqslant \lim _{j} \inf \lim \inf _{i}\left\|z_{i}-z_{j}\right\|$. Consequently, we get $\beta_{1}-\beta_{2} \leqslant \operatorname{csep}\left(z_{k}\right)+\varepsilon \leqslant \gamma(A)+\varepsilon$. Hence $\gamma(A)+\varepsilon \geqslant e(A)$ and finally $\gamma(A) \geqslant e(A)$.

2.2. $\gamma$ AND DE BLASI'S MEASURE $\omega$ The axiomatic approach to a measure of weak noncompactness, presented in subsection 2.1, was preceded by a definition of the measure $\omega$ introduced by De Blasi (see [16] and the references given there). For a nonempty and bounded subset $A$ of a Banach space $X$ let

$$
\omega(A)=\inf \left\{t>0: A \subset C+t \overrightarrow{\mathrm{B}}_{X} \text { and } C \subset X \text { is a weakly compact set }\right\} .
$$

Theorem 2.5 and a result of Astala and Tylli (see [4]) show that, in general, the measures $\gamma$ and $\omega$ are not equivalent. Certainly, if $X$ is reflexive, then $\bar{B}_{X}$ is weakly compact and $\omega\left(\bar{B}_{X}\right)=0$. Otherwise it is shown $[16]$ that $\omega\left(\bar{B}_{X}\right)=1$. Similarly, for reflexive spaces $\gamma\left(\bar{B}_{X}\right)=0$. The nonreflexive case for $\gamma$ differs from the one for $\omega$.

EXAMPLE 2.6. Consider the vectors $e_{n}=(0, \ldots, 0,1,0, \ldots) \in l_{1}, n \in \mathbb{N}$, where 1 occupies the $n$-th place. It is evident that $\operatorname{csep}\left(e_{n}\right)=2$ and therefore $\gamma\left(\bar{B}_{l_{1}}\right)=2$.

EXAMPLE 2.7. The case of $c_{0}$ is more complicated. From the next theorem we shall see at once that $\gamma\left(\bar{B}_{c_{0}}\right)=1$.

ThEOREM 2.8. Let $A$ be a nonempty and bounded subset in $c_{0}$. Then

$$
\gamma(A)=\sup \operatorname{dist}\left(x^{* *}, c_{0}\right)
$$

where the supremum is taken over all sequences $\left(x_{n}\right)$ in conv $A$ and all $w^{*}$-cluster points $x^{* *} \in c_{0}^{* *}$ of $\left(x_{n}\right)$.

ProOF: Let us denote by $\gamma^{\prime}(A)$ the right-hand side of formula (2.3). In view of Theorem 2.4 it is enough to prove that $\gamma(A) \leqslant \gamma^{\prime}(A)$. Choose $M$ such that $A \subset M \overline{\mathrm{B}}_{c_{0}}$ and fix a sequence $\left(x_{n}\right) \subset$ conv $A$. For each $w^{*}$-cluster point $x^{* *}=\left(x^{* *}(k)\right)$ of $\left(x_{n}\right)$ there exists a subsequence $\left(x_{n_{i}}\right)$ such that $x^{* *}=w^{*}-\lim _{i \rightarrow \infty} x_{n_{i}}$. Let $q=\operatorname{dist}\left(x^{* *}, c_{0}\right)=\limsup _{k \rightarrow \infty}\left|x^{* *}(k)\right|$ and $y_{i}=x_{n_{i}}$ for $i \in \mathbb{N}$. Fix $\varepsilon>0$ and $N \in \mathbb{N}$. Let us choose a subsequence $\left(y_{n_{i}}\right)_{i=1}^{N}$ and an increasing sequence $\left(k_{i}\right)_{i=1}^{N+1}$ of natural numbers such that $\left|x^{* *}(k)\right|<q+\varepsilon$ for $k>k_{1}$, and satisfying the following conditions: $\left|y_{n_{i}}(k)-x^{* *}(k)\right|<\varepsilon$ for $k \leqslant k_{i}$ and $\left|y_{n_{i}}(k)\right|<\varepsilon$ for $k>k_{i+1}, i=1, \ldots, N$. Let us define $x=(1 / N) \sum_{i=1}^{N} y_{n_{i}} \in \operatorname{conv}\left(x_{n}\right)$. Then for $k>k_{1}$ 
we have

$$
\left|x(k)-x^{* *}(k)\right|<\left|x^{* *}(k)\right|+\varepsilon+\frac{M}{N}<q+2 \varepsilon+\frac{M}{N}
$$

and for $k \leqslant k_{1}$

$$
\left|x(k)-x^{* *}(k)\right|<\varepsilon .
$$

Both the inequalities and an arbitrary choice of $\varepsilon$ and $N$ yields dist $\left(x^{* *}, \operatorname{conv}\left(x_{n}\right)\right) \leqslant q$ and hence $\gamma(A) \leqslant \gamma^{\prime}(A)$.

THEOREM 2.9. The measures $\gamma$ and $\omega$ are equal in $c_{0}$.

Proof: Given $q \geqslant 0$, we set

$$
r_{q}(\alpha)=\left\{\begin{array}{lll}
0 & \text { if } & |\alpha| \leqslant q \\
\alpha-q \frac{\alpha}{|\alpha|} & \text { if } & |\alpha|>q .
\end{array}\right.
$$

Next, let $R_{q}: c_{0} \rightarrow c_{0}$ be given by the formula $R_{q} x=\left(r_{q}(x(k))\right)$, where $x=(x(k))$. We take a nonempty bounded set $A \subset c_{0}$ and put $q=\gamma(A)$. We shall show that $\gamma\left(R_{q}(A)\right)=0$. Let $\left(x_{n}\right) \subset A$ and suppose that the limit $w^{*} \lim _{n \rightarrow \infty} x_{n}=x^{* *} \in c_{0}^{* *}$ exists. By Theorem $2.8, \limsup _{k \rightarrow \infty}\left|x^{* *}(k)\right| \leqslant q$. Therefore

$$
\limsup _{k \rightarrow \infty}\left|\left(w^{*}-\lim _{n \rightarrow \infty} R_{q} x_{n}\right)(k)\right|=\limsup _{k \rightarrow \infty}\left|r_{q}\left(x^{* *}(k)\right)\right|=r_{q}\left(\limsup _{k \rightarrow \infty}\left|x^{* *}(k)\right|\right)=0 .
$$

In view of Theorem 2.8 we obtain $\gamma\left(R_{q}(A)\right)=0$. It follows that $R_{q}(A)$ is relatively weakly compact. Since $A \subset R_{q}(A)+q \overline{\mathrm{B}}_{c_{0}}$, we get $\omega(A) \leqslant q=\gamma(A)$.

On the other hand, we have $\gamma(A) \leqslant \gamma\left(\overline{\mathrm{B}}_{c_{0}}\right) \omega(A)=\omega(A)$ (see [7]).

\section{REAL INTERPOLATION OF OPERATORS}

3.1. MEASURE OF WEAK NONCOMPACTNESS FOR OPERATORS By $L(X, Y)$ we denote the space of all linear and bounded operators $T: X \rightarrow Y$ between Banach spaces $X$ and $Y$. Using the measure $\gamma$, introduced in the previous section, we can define the measure of weak noncompactness for every operator $T \in L(X, Y)$. This measure provides in addition a seminorm in $L(X, Y)$.

DEFinition 3.1: For every $T \in L(X, Y)$ the number $\Gamma(T)=\gamma\left(T\left(\mathrm{~B}_{X}\right)\right)$ is called the measure of weak noncompactness of the operator $T$.

Let $X$ be a Banach space. We denote by $l_{p}(X), 1<p<\infty$ the Banach space of all sequences $x=(x(i))$ such that $x(i) \in X$ for all $i \in \mathbb{N}$ and $\|x\|_{l_{p}(X)}=\left(\sum_{i=1}^{\infty}\|x(i)\|^{p}\right)^{1 / p}$ is 
finite. A standard verification shows that we can identify $\left(l_{p}(X)\right)^{*}$ with $l_{q}\left(X^{*}\right)$, where $1 / p+1 / q=1$, and for each $\phi=(\phi(i)) \in\left(l_{p}(X)\right)^{*}$ we have $\phi(x)=\sum_{i=1}^{\infty} \phi(i)(x(i))$ for all $x \in l_{p}(X)$ (see [21]). If $X, Y$ are Banach spaces and $T \in L(X, Y)$, then the operator $\widetilde{T}: l_{p}(X) \rightarrow l_{p}(Y)$ given by $\widetilde{T} x=(T x(i))$ is also bounded and $\|\widetilde{T}\|=\|T\|$.

To deal with the measure $\gamma$, ultrafilters will be used in several cases. For more details concerning filters we refer the reader for instance to [2]. We recall two important facts.

LEMMA 3.2. Let $\mathcal{U}$ be an ultrafilter on a nonempty set $I$ and $I_{1} \cup I_{2}=I$. Then $I_{1}$ or $I_{2}$ belongs to $\mathcal{U}$.

LEMMA 3.3. An ultrafilter $\mathcal{U}$ is free if and only if $\mathcal{U}$ does not contain a finite set.

Let $\tau$ be a Hausdorff topology in a space $E$ and let $\mathcal{U}$ be an ultrafilter on the set of positive integers $\mathbb{N}$. An element $x \in E$ is said to be the limit over $\mathcal{U}$ of a sequence $\left(x_{n}\right)$ in $E$, if $\left\{n \in \mathbb{N}: x_{n} \in V\right\} \in \mathcal{U}$ for every neighbourhood $V$ of $x$. Then we write $x=\tau$ - $\lim _{\mathcal{U}} x_{n}$ or simply $x=\lim _{\mathcal{u}} x_{n}$. Let us recall that if $E$ is compact, then $\lim _{\mathcal{u}} x_{n}$ exists for each sequence $\left(x_{n}\right)$ in $E$.

The next lemma, roughly speaking, can stand for a passing to a subsequence. In the light of Lemma 3.3, the proof is straightforward.

LEMMA 3.4. Let $\mathcal{U}$ be a free ultrafilter on $\mathbb{N}$ and $N_{1} \in \mathcal{U}$. If $f: N_{1} \rightarrow \mathbb{N}$ is the bijection given by $f\left(n_{k}\right)=k$, then $\mathcal{U}_{1}=\left\{f\left(N_{1} \cap A\right): A \in \mathcal{U}\right\}$ is a free ultrafilter on $\mathbb{N}$. Moreover, if $\lim _{\mathcal{U}} x_{n}=x$ in a topological space $X$, then $\lim _{\mathcal{u}_{1}} x_{n_{k}}=x$ as well.

LEMMA 3.5. Let $Y$ be a Banach space and $1<p<\infty$. If $y=(y(i)), y_{n}=$ $\left(y_{n}(i)\right) \in l_{p}\left(Y^{* *}\right)$ for all $n \in \mathbb{N}$ and $y=w^{*}-\lim _{u} y_{n}$ over some free ultrafilter $\mathcal{U}$ on $\mathbb{N}$, then $y(i)=w^{*}-\lim _{u} y_{n}(i)$ for each $i \in \mathbb{N}$.

ProOF: Let us first recall that if $f: X \rightarrow Y$ is a continuous function between topological spaces, $\left(x_{i}\right)_{i \in I} \subset X$ and $x=\lim _{\mathcal{U}^{\prime}} x_{i}$ over a free ultrafilter $\mathcal{U}^{\prime}$ on a set of indices $I$, then $f(x)=\lim _{\mathcal{U}^{\prime}} f\left(x_{i}\right)$. Fix $i \in \mathbb{N}$ and take $v=(0, \ldots, 0, v(i), 0, \ldots) \in l_{q}\left(Y^{*}\right)$. The functional $f_{v}$ given by the formula $f_{v}(z)=z(v)=z(i)(v(i))$ for $z \in l_{p}\left(Y^{* *}\right)$ is $w^{*}$-continuous. Consequently, $f_{v}\left(y_{n}\right)=y_{n}(i)(v(i))$ and $y(i)(v(i))=f_{v}(y)=w^{*}-\lim _{u} y_{n}(i)(v(i))$ for every $v(i) \in Y^{*}$, which is equivalent to $y(i)=w^{*}-\lim _{u} y_{n}(i)$.

Theorem 3.6. Let $X, Y$ be Banach spaces and $1<p<\infty$. If $T \in L(X, Y)$ and $\widetilde{T} \in L\left(l_{p}(X), l_{p}(Y)\right)$ is defined by $\tilde{T} x=(T x(i))$ for $x=(x(i)) \in l_{p}(X)$, then $\Gamma(T)=\Gamma(\widetilde{T})$.

ProOF: Since $T=\tilde{T} \mid\{(x, 0,0, \ldots)\}$, we see that $\Gamma(T) \leqslant \Gamma(\tilde{T})$. For the proof of $\Gamma(T) \geqslant \Gamma(\widetilde{T})$, assume that $\Gamma(\widetilde{T})>0$, otherwise at once $\Gamma(T)=\Gamma(\widetilde{T})$. Fix $0<\varepsilon<\Gamma(\widetilde{T})$ and let $\Gamma_{1}=\Gamma(\tilde{T})-\varepsilon$. There exists $\left(x_{n}\right) \subset \mathrm{B}_{l_{p}(X)}$ such that for $y_{n}=\tilde{T} x_{n}$ we have $0<\Gamma_{1} \leqslant \operatorname{dist}\left(y, \operatorname{conv}\left(y_{n}\right)\right) \leqslant \operatorname{dist}\left(y,\left(y_{n}\right)\right)$, where $y \in l_{p}\left(Y^{* *}\right)$ is a $w^{*}$-cluster point of 
the sequence $\left(y_{n}\right)$ and therefore $y=w^{*}$ - $\lim _{u} y_{n}$ over some free ultrafilter $\mathcal{U}$ in $\mathbb{N}$. By a separation theorem there exists $\phi \in l_{q}\left(Y^{* * *}\right)$ such that $\|\phi\| \leqslant 1$ and $\phi(z) \geqslant \Gamma_{1}$ for all $z \in y-\operatorname{conv}\left(y_{n}\right)$. Now, fix $m \in \mathbb{N}$ satisfying $\sum_{i=m+1}^{\infty}\|\phi(i)\|^{q}<\varepsilon^{q}$. The boundedness of $y-\operatorname{conv}\left(y_{n}\right)$ gives a constant $c>0$ such that

$$
\begin{aligned}
\Gamma_{1} & \leqslant \sum_{i=1}^{m} \phi(i)\left(y(i)-y_{n}(i)\right)+\left(\sum_{i=m+1}^{\infty}\|\phi(i)\|^{q}\right)^{1 / q}\left(\sum_{i=m+1}^{\infty}\left\|y(i)-y_{n}(i)\right\|^{p}\right)^{1 / p} \\
& \leqslant \sum_{i=1}^{m} \phi(i)\left(y(i)-y_{n}(i)\right)+\varepsilon c
\end{aligned}
$$

for every $n \in \mathbb{N}$, where, by Lemma $3.5, y(i)=w^{*}-\lim _{u} y_{n}(i)$ for all $i$. Writing $I=\{1 \leqslant$ $i \leqslant m: \phi(i) \neq 0\}$ and

$$
\psi(i)=\frac{\phi(i)}{\|\phi(i)\|} \text { for } i \in I, \quad \alpha_{i}=\lim _{\mathcal{U}}\left\|x_{n}(i)\right\|, \quad v(i)=\frac{y(i)}{\alpha_{i}+(\varepsilon / m)}, \quad v_{n}(i)=\frac{y_{n}(i)}{\alpha_{i}+(\varepsilon / m)},
$$

by Hölder's and Minkowski's inequalities we obtain

$$
\begin{aligned}
\Gamma_{1}-\varepsilon c & \leqslant \sum_{i \in I}\|\phi(i)\|\left(\alpha_{i}+\frac{\varepsilon}{m}\right) \psi(i)\left(v(i)-v_{n}(i)\right) \\
& \leqslant\left(\sum_{i=1}^{m}\|\phi(i)\|^{q}\right)^{1 / q}\left(\sum_{i=1}^{m}\left(\alpha_{i}+\frac{\varepsilon}{m}\right)^{p}\right)^{1 / p} \max _{i \in I} \psi(i)\left(v(i)-v_{n}(i)\right) \\
& \leqslant\left(1+\varepsilon m^{1 / p-1}\right) \max _{i \in I} \psi(i)\left(v(i)-v_{n}(i)\right) .
\end{aligned}
$$

We set $\Gamma_{2}=\left(\Gamma_{1}-\varepsilon c\right)\left(1+\varepsilon m^{1 / p-1}\right)^{-1}$. Then

$$
\Gamma_{2} \leqslant \max _{i \in I} \psi(i)\left(v(i)-v_{n}(i)\right)
$$

for all $n \in \mathbb{N}$. For each $1 \leqslant i \leqslant m$ let $N_{i}$ denote the set of all natural numbers $n$ for which the maximum in (3.1) is attained for $i$. It is clear that $\bigcup_{1 \leqslant i \leqslant m} N_{i}=\mathbb{N}$ and, by Lemma 3.2 , $N_{j} \in \mathcal{U}$ for some $j$. Let us apply Lemma 3.4 for the set $N_{j} \cap\left\{n \in \mathbb{N}:\left|\left\|x_{n}(j)\right\|-\alpha_{j}\right|<\right.$ $\varepsilon / m\}=\left\{n_{k}\right\} \in \mathcal{U}$ and change $\mathcal{U}$ to a free ultrafilter $\mathcal{U}_{1}$ as given by this lemma. It follows that

$$
\Gamma_{2} \leqslant \psi(j)\left(v(j)-v_{n_{k}}(j)\right)
$$

for $k \in \mathbb{N}$. Considering convex combinations of the obtained sequence yields

$$
\Gamma_{2} \leqslant \operatorname{dist}\left(v(j), \operatorname{conv}\left(v_{n_{k}}(j)\right)\right)
$$

But $\left(v_{n_{k}}(j)\right) \subset T\left(\mathrm{~B}_{X}\right)$ and $v(j)=w^{*}-\lim _{u_{1}} v_{n_{k}}(j)$, which gives $\Gamma_{2} \leqslant \Gamma(T)$. By letting $\varepsilon \rightarrow 0$ we conclude that $\Gamma(\tilde{T}) \leqslant \Gamma(T)$ and this finishes the proof. 
REMARK 3.7. We can consider a space $l_{p}(X)$ of sequences indexed by the set of all integers $\mathbb{Z}$. The same properties as stated at the beginning of this section, Lemma 3.5 and Theorem 3.6 also hold in that case.

3.2. Real interpolation We shall say that Banach spaces $A_{0}$ and $A_{1}$ are compatible if they are continuously embedded in a Hausdorff topological vector space $X$. Then $A_{0} \cap A_{1}$ and $A_{0}+A_{1}$ with norms

$$
\|a\|_{A_{0} \cap A_{1}}=\max \left\{\|a\|_{A_{0}},\|a\|_{A_{1}}\right\} \text { and }\|a\|_{A_{0}+A_{1}}=\inf _{a=a_{0}+a_{1}}\left\{\left\|a_{0}\right\|_{A_{0}}+\left\|a_{1}\right\|_{A_{1}}\right\}
$$

respectively, are also Banach spaces. Let $\bar{A}=\left(A_{0}, A_{1}\right)$ denote a pair of compatible Banach spaces $A_{0}$ and $A_{1}$. A Banach space $A$ is said to be an intermediate space with respect to $\bar{A}$ if

$$
A_{0} \cap A_{1} \subset A \subset A_{0}+A_{1}
$$

and both inclusions are continuous. Let $\bar{A}=\left(A_{0}, A_{1}\right)$ and $\bar{B}=\left(B_{0}, B_{1}\right)$ be two pairs of compatible Banach spaces and $T$ a linear operator from $A_{0}+A_{1}$ into $B_{0}+B_{1}$. We write $T: \bar{A} \rightarrow \bar{B}$ for brevity, if $T \in L\left(A_{0}, B_{0}\right)$ and $T \in L\left(A_{1}, B_{1}\right)$, viewed as the restrictions of $T$. If $A$ and $B$ are intermediate spaces with respect to $\bar{A}$ and $\bar{B}$ respectively, and $T: \bar{A} \rightarrow \bar{B}$ implies $T \in L(A, B)$, then $A$ and $B$ are said to be interpolation spaces with respect to $\bar{A}$ and $\bar{B}$.

In the sequel, we restrict our considerations to one of the equivalent constructions of so called real interpolation spaces, that is, a discrete method introduced by Lions and Peetre [21]. Let $0<\theta<1,1<p<\infty$ and for each $a \in A_{0}+A_{1}$

$$
\|a\|_{\theta, p}=\inf \max \left\{\left\|\left(2^{i \theta} a_{0}(i)\right)\right\|_{l_{p}\left(A_{0}\right)},\left\|\left(2^{i(\theta-1)} a_{1}(i)\right)\right\|_{l_{p}\left(A_{1}\right)}\right\}
$$

where the infimum is taken over all $\left(a_{0}(i)\right) \subset A_{0},\left(a_{1}(i)\right) \subset A_{1}$ such that $a_{0}(i)+a_{1}(i)=a$ for all $i \in \mathbb{Z}$. Then $A_{\theta, p}=\left\{a \in A_{0}+A_{1}:\|a\|_{\theta, p}<\infty\right\}$ is an intermediate space with respect to $\bar{A}$ and moreover one can show [21] that for $a \in A_{\theta, p}$

$$
\|a\|_{\theta, p} \leqslant 2^{\theta(1-\theta)} \inf _{a_{0}(i)+a_{1}(i)=a}\left\|\left(2^{i \theta} a_{0}(i)\right)\right\|_{l_{p}\left(A_{0}\right)}^{1-\theta}\left\|\left(2^{i(\theta-1)} a_{1}(i)\right)\right\|_{l_{p}\left(A_{1}\right)}^{\theta} .
$$

The spaces $A_{\theta, p}$ and $B_{\theta, p}$, obtained by this method, are interpolation spaces with respect to $\bar{A}$ and $\bar{B}$. Furthermore, for every $T: \bar{A} \rightarrow \bar{B}$

$$
\|T\|_{\theta, p} \leqslant 2^{\theta(1-\theta)}\|T\|_{0}^{1-\theta}\|T\|_{1}^{\theta}
$$

where $\|T\|_{\theta, p}$ and $\|T\|_{i}, i=0,1$ are norms of operators $T: A_{\theta, p} \rightarrow B_{\theta, p}$ and $T: A_{i} \rightarrow B_{i}$, $i=0,1$ respectively.

We prove an analogous inequality for our measure of weak noncompactness for operators. Note that a similar result for De Blasi's measure does not hold (see [12]). 
THEOREM 3.8. Let $A_{\theta, p}$ and $B_{\theta, p}$ be interpolation spaces with respect to $\bar{A}=$ $\left(A_{0}, A_{1}\right)$ and $\bar{B}=\left(B_{0}, B_{1}\right)$ obtained by the real method described above for some $0<$ $\theta<1$ and $1<p<\infty$. Then for every $T: \bar{A} \rightarrow \bar{B}$

$$
\Gamma_{\theta, p}(T) \leqslant 2^{\theta(1-\theta)} \Gamma_{0}(T)^{1-\theta} \Gamma_{1}(T)^{\theta},
$$

where $\Gamma_{\theta, p}$ and $\Gamma_{i}, i=0,1$ are measures of weak noncompactness for operators $T: A_{\theta, p} \rightarrow$ $B_{\theta, p}$ and $T: A_{i} \rightarrow B_{i}, i=0,1$ respectively.

PROOF: Fix $\varepsilon>0$ and a sequence $\left(a_{n}\right) \subset \mathrm{B}_{A_{\theta, \mathrm{p}}}$. For each $a_{n}$ there exist

$$
\left(2^{i \theta} a_{0 n}(i)\right)_{i \in \mathbf{Z}} \in \mathrm{B}_{l_{p}\left(A_{0}\right)} \text { and }\left(2^{i(\theta-1)} a_{1 n}(i)\right)_{i \in \mathbf{Z}} \in \mathrm{B}_{l_{p}\left(A_{1}\right)}
$$

such that $a_{0 n}(i)+a_{1 n}(i)=a_{n}$ for all $i \in \mathbb{Z}$. Let $y_{n}=\left(2^{i \theta} T a_{0 n}(i)\right)_{i \in \mathbf{Z}}, z_{n}=$ $\left(2^{i(\theta-1)} T a_{1 n}(i)\right)_{i \in \mathbf{Z}}$ and $b_{n}=T a_{n}$ for every $n \in \mathbb{N}$. By a similar method to that used in the proof of condition (5) of Theorem 2.3, we can obtain sequences $\left(y_{n}^{\prime \prime}\right),\left(z_{n}^{\prime \prime}\right)$ of $s c c$ for $\left(y_{n}\right),\left(z_{n}\right)$ respectively. They satisfy therefore the assertion of Theorem 2.1, $y_{k}^{\prime \prime}=\sum_{j=n_{k}+1}^{n_{k+1}} \lambda_{j}^{k} y_{j}$ and $z_{k}^{\prime \prime}=\sum_{j=n_{k}+1}^{n_{k+1}} \lambda_{j}^{k} z_{j}$ for some $0=n_{1}<n_{2}<n_{3}<\ldots$ and some nonnegative coefficients $\lambda_{n_{k}+1}^{k}, \ldots, \lambda_{n_{k+1}}^{k}$ with $\sum_{j=n_{k}+1}^{n_{k+1}} \lambda_{j}^{k}=1$. We set $b_{k}^{\prime \prime}=\sum_{j=n_{k}+1}^{n_{k+1}} \lambda_{j}^{k} b_{j}$ for $k=1,2$. Then

$$
\operatorname{csep}\left(b_{n}\right) \leqslant\left\|b_{1}^{\prime \prime}-b_{2}^{\prime \prime}\right\|_{\theta, p} \leqslant 2^{\theta(1-\theta)}\left\|y_{1}^{\prime \prime}-y_{2}^{\prime \prime}\right\|_{l_{p}\left(B_{0}\right)}^{1-\theta}\left\|z_{1}^{\prime \prime}-z_{2}^{\prime \prime}\right\|_{l_{p}\left(B_{1}\right)}^{\theta},
$$

the last inequality being a consequence of (3.2). But $\left\|y_{1}^{\prime \prime}-y_{2}^{\prime \prime}\right\|_{l_{p}\left(B_{0}\right)} \leqslant \operatorname{csep}\left(y_{n}^{\prime \prime}\right)+\varepsilon$ and $\left\|z_{1}^{\prime \prime}-z_{2}^{\prime \prime}\right\|_{l_{p}\left(B_{1}\right)} \leqslant \operatorname{csep}\left(z_{n}^{\prime \prime}\right)+\varepsilon$. Moreover, $y_{n}^{\prime \prime} \in \tilde{T}_{0}\left(\mathrm{~B}_{l_{p}\left(A_{0}\right)}\right)$ and $z_{n}^{\prime \prime} \in \tilde{T}_{1}\left(\mathrm{~B}_{l_{p}\left(A_{1}\right)}\right)$ for all $n \in \mathbb{N}$, where $\widetilde{T}_{i}: l_{p}\left(A_{i}\right) \rightarrow l_{p}\left(B_{i}\right), i=0,1$ is defined as in Theorem 3.6. Hence

$$
\operatorname{csep}\left(b_{n}\right) \leqslant 2^{\theta(1-\theta)}\left(\Gamma\left(\widetilde{T}_{0}\right)+\varepsilon\right)^{1-\theta}\left(\Gamma\left(\widetilde{T}_{1}\right)+\varepsilon\right)^{\theta} .
$$

Finally, Theorem 3.6 with Remark 3.7 and an arbitrary choice of $\varepsilon$ and $\left(a_{n}\right)$ lead to the desired conclusion.

Since $\Gamma(T)=0$ if and only if $T$ is weakly compact, the above theorem brings a new proof of the following: if $T: A_{0} \rightarrow B_{0}$ or $T: A_{1} \rightarrow B_{1}$ is weakly compact, then so is $T: A_{\theta, p} \rightarrow B_{\theta, p}$ for all $0<\theta<1$ and $1<p<\infty$. Let us also formulate another immediate consequence of Theorem 3.8.

Corollary 3.9. Let $0<\theta<1,1<p<\infty$ and let $A_{\theta, p}$ be the interpolation space with respect to $\bar{A}=\left(A_{0}, A_{1}\right)$. Then

$$
\gamma\left(\mathrm{B}_{A_{\theta, p}}\right) \leqslant 2^{\theta(1-\theta)} \gamma\left(\mathrm{B}_{A_{0}}\right)^{1-\theta} \gamma\left(\mathrm{B}_{A_{1}}\right)^{\theta} .
$$

In particular, if $A_{0}$ or $A_{1}$ is reflexive then $A_{\theta, p}$ is reflexive as well. 


\section{REFERENCES}

[1] R.R. Akhmerov, M.I. Kamenskii, A.S. Potapov, A.E. Rodkina and B.N. Sadovskii, Measures of noncompactness and condensing operators (Birkhäuser Verlag, Basel, Boston, Berlin, 1992).

[2] A.G. Aksoy and M.A. Khamsi, Nonstandard methods in fixed point theory (Springer-Verlag, Berlin, Heidelberg, New York, 1990).

[3] A.G. Aksoy and L. Maligranda, 'Real interpolation and measure of weak noncompactness', Math. Nachr. 175 (1995), 5-12.

[4] K. Astala and H.-O. Tylli, 'Seminorms related to weak compactness and to Tauberian operators', Math. Proc. Cambridge Philos. Soc. 107 (1990), 367-375.

[5] J. Banaś, 'Applications of measures of weak noncompactness and some classes of operators in the theory of functional equations in the Lebesgue space', (Proceedings of the Second World Congress of Nonlinear Analysts, Part 6, Athens, 1996), Nonlinear Anal. 30 (1997), 3283-3293.

[6] J. Banaś and A. Martinón, 'Measures of weak noncompactness in Banach sequence spaces', Portugal. Math. 52 (1995), 131-138.

[7] J. Banaś and J. Rivero, 'On measures of weak noncompactness', Ann. Mat. Pura Appl. 151, 213-224.

[8] B. Beauzamy, Espaces d'interpolation réels: topologie et géométrie (Springer-Verlag, Berlin, Heidelberg, New York, 1978).

[9] Yu.A. Brudnyi and N.Ya. Krugljak, Interpolation functors and interpolation spaces. Vol. I (North-Holland, Amsterdam, New York, Oxford, Tokyo, 1991).

[10] F. Cobos, Interpolation theory and measures related to operator ideals, (M. Krbec and A. Kufner, Editors), Nonlinear analysis, function spaces and applications, Vol. 6 (Olympia Press, Prague, 1999).

[11] F. Cobos, P. Fernández-Martínez and A. Martínez, 'Interpolation of the measure of non-compactness by the real method', Studia Math. 135 (1999), 25-38.

[12] F. Cobos, A. Manzano and A. Martínez, 'Interpolation theory and measures related to operator ideals', Quart. J. Math. Oxford Ser. (2) 50 (1999), 401-416.

[13] F. Cobos and A. Martínez, 'Extreme estimates for interpolated operators by the real method', J. London Math. Soc. (2) 60 (1999), 860-870.

[14] F. Cobos and A. Martínez, 'Remarks on interpolation properties of the measure of weak non-compactness and ideal variations', Math. Nachr. 208 (1999), 93-100.

[15] M. Cwikel, 'Real and complex interpolation and extrapolation of compact operators', Duke Math. J. 65 (1992), 333-343.

[16] F.S. De Blasi, 'On a property of the unit sphere in a Banach space', Bull. Math. Soc. Sci. Math. R.S. Roumanie 21 (1977), 259-262.

[17] D. van Dulst, Reflexive and superreflexive Banach spaces (Mathematisch Centrum, Amsterdam, 1978).

[18] S. Heinrich, 'Closed operator ideals and interpolation', J. Funct. Anal. 35 (1980), 397-411.

[19] R.C. James, 'Weak compactness and reflexivity', Israel J. Math. 2 (1964), 101-119.

[20] M.A. Krasnoselskii, 'On a theorem of M. Riesz', (Russian), Dokl. Akad. Nauk SSSR 131 (1960), 246-248. 
[21] J.-L. Lions and J. Peetre, 'Sur une classe d'espaces d'interpolation', Inst. Hautes Études Sci. Publ. Math. 19 (1964), 5-68.

[22] L. Maligranda and A. Quevedo, 'Interpolation of weakly compact operators', Arch. Math. 55 (1990), 280-284.

[23] M. Mastylo, 'On interpolation of weakly compact operators', Hokkaido Math. J. 22 (1993), 105-114.

[24] S. Mazur, 'Über konvexe Mengen in linearen normierten Räumen', Studia Math. 4 (1933), 70-84.

[25] D.P. Milman and V.D. Milman, 'The geometry of imbeddings with empty intersection. The structure of the unit sphere in a non-reflexive space', (Russian), Mat. Sbornik 66 (1965), 109-118.

[26] V.D. Milman, 'Geometric theory of Banach spaces. II. Geometry of the unit ball', (Russian), Uspekhi Mat. Nauk 26 (1971), 73-149. English translation: Russian Math. Surv. 26 (1971), 79-163.

[27] M.F. Teixeira and D.E. Edmunds, 'Interpolation theory and measures of non-compactness', Math. Nachr. 104 (1981), 129-135.

Institute of Mathematics

Maria Curie-Skłodowska University

20-031 Lublin

Poland

e-mail: akryczka@golem.umcs.lublin.pl

bsprus@golem.umcs.lublin.pl

szczepan@golem.umcs.lublin.pl 